\title{
À la recherche d'une base conceptuelle solide de la biologie dans l'éducation à la santé
}

Aliana Fernandes-Guimaräes et Mercedes Manzanares-Gavilan

\section{(2) OpenEdition \\ 1 Journals}

Édition électronique

URL : http://journals.openedition.org/trema/2023

DOI : $10.4000 /$ trema.2023

ISSN : 2107-0997

\section{Éditeur}

Faculté d'Éducation de l'université de Montpellier

\section{Édition imprimée}

Date de publication : 1 septembre 1996

Pagination : 73-80

ISSN : 1167-315X

\section{Référence électronique}

Aliana Fernandes-Guimaräes et Mercedes Manzanares-Gavilan, «À la recherche d'une base conceptuelle solide de la biologie dans l'éducation à la santé », Tréma [En ligne], 9-10 | 1996, mis en ligne le 11 septembre 2013, consulté le 19 avril 2019. URL : http://journals.openedition.org/ trema/2023 ; DOI : 10.4000/trema.2023

Ce document a été généré automatiquement le 19 avril 2019.

Trema 


\title{
À la recherche d'une base conceptuelle solide de la biologie dans l'éducation à la santé
}

\author{
Aliana Fernandes-Guimaräes et Mercedes Manzanares-Gavilan
}

\section{Introduction}

1 La santé est un concept multidimensionnel, qui concerne autant l'état physique que le mental et le social. La seule introduction des contenus académiques traditionnels ne fournit pas une base solide dans les énoncés de l'EAS (Éducation à la santé), sans base conceptuelle en biologie. Cela rend évident le besoin d'aborder de façon transversale ce domaine éducatif.

2 Nous considérons donc, tout d'abord, qu'on doit avoir la base conceptuelle scientifique, parce qu'elle touche presque totalement l'aspect physique de la santé qui, comme on le verra ensuite, est la perception la plus claire que nos élèves ont sur la santé.

On doit considérer les postulats de Ausubel (1978) et de Novak (1982) à propos du modèle d'apprentissage significatif. Ils soutiennent, grosso modo, cette idée : l'élève construit sa propre pensée, puis il la contraste avec son expérience et il la restructure, en fonction de la propre structure qu'il avait et du résultat de l'interaction entre celle-ci et le nouvel apprentissage. L'apprentissage significatif se produira uniquement si la connexion avec sa structure préalable se réalise de façon adéquate.

4 D'autre part, des études ont mis en évidence l'importance et la persistance des idées préalables des élèves dans le processus enseigner. Ceci a conduit à la réalisation d'une thèse qui défend que pour que cet apprentissage se réalise, il est nécessaire qu'il se produise le changement conceptuel des idées préalables pour les nouvelles idées (Posner, 1992), et pour certains concepts, un changement méthodologique est nécessaire (Gil et al., 1985). Toutes ces contributions ont conduit à reconsidérer les méthodologies d'enseignement, et notamment celles qu'on pourrait considérer comme dominées par une optique constructiviste (Driver et al., 1986). 
5 Dans le cas de l'EAS, Prout (1985) a mis en évidence l'importance du savoir non scientifique ou quotidien dans les clichés d'EAS, en soulignant que la persistance des préconceptions est due aux pratiques culturelles et au grand pouvoir d'explication pour les expériences de chaque jour, même au-delà de celles qui proviennent des connaissances biologiques. De ce point de vue, le savoir scientifique est désavantagé par rapport aux connaissances des sujets en EAS.

\section{Les représentations des élèves}

\subsection{Alimentation et nutrition}

6 Il y a une grande diversité de conceptions, nombre d'entre elles sont en rapport étroit avec les perceptions individuelles et les croyances et messages familiaux.

7 Les réponses des élèves montrent une confusion entre aliment et nutriment. Ils considèrent comme aliment n'importe quel produit qui entre par la bouche dans notre organisme, ils associent de même l'aliment avec des produits capables d'assouvir leur faim.

Généralement le concept de nutrition qu'apportent les élèves rejoint celui de la digestion, et, comme on sait, il ne constitue qu'une des fonctions de la digestion. Cela est dû à une conception "organique » de la nutrition qui n'envisage pas les processus cellulaires ou métaboliques. La nutrition est conçue comme un simple processus de réduction du volume des substances alimentaires pour nourrir le corps (cette dernière idée étant floue et imprécise). Dans ce modèle explicatif, la circulation n'est pas nécessaire (l'aliment digéré se répand directement aux organes), ni la respiration, ni le métabolisme (on pense que cela a été déjà fait par la digestion).

D'autre part, Mintzes (1984) a constaté par ses études que les représentations du substrat anatomique dans lequel ces fonctions se développent, se caractérisent par une grandeur disproportionnée des organes (ex: les poumons sont deux sacs trop petits), un emplacement inadéquat et une absence de relation entre eux (ex: il n'y a pas de connexion entre les systèmes digestifs et circulatoires).

Cubero (1986) constate l'omission de certains organes (ex : on oublie fréquemment le foie et le pancréas dans l'appareil digestif). Un autre type de représentation relève d'une conception incomplète de la nutrition. Ainsi, par exemple, la connexion entre digestif et excréteur, témoignée par de nombreux auteurs (Giordan, 1987 ; Banet, 1988) suppose, à notre avis, l'évacuation de produits liquides séparément de celle qui se réalise par la voie digestive : l'excrétion n'est pas l'élimination des produits du métabolisme cellulaire, mais de la propre digestion.

En ce qui concerne les processus physiologiques de la digestion, nous sommes d'accord avec Banet (1988), qui a mis en évidence une notion simpliste selon laquelle la digestion consiste à la simple séparation de "bonnes et mauvaises" substances. Nous avons constaté, de même, une idée de la digestion tout à fait mécanique, dans laquelle le foie et le pancréas n'ont aucun rôle. Toutes ces idées mettent en évidence l'absence d'un modèle chimique de la digestion et, en rapport avec cela, une notion primitive de la nature de la matière (dans ce cas-là des aliments) et de ses transformations. Ces difficultés ne sont qu'une partie d'une idée déformée de l'anatomie et la physiologie, ainsi que de la fonction de relation. 


\subsection{Activité physique} sang au moyen de l'exercice physique ne sont pas connus par ces étudiants. Pour eux, tout exercice effectué par un individu a le même but ; autrement dit, les différentes sortes d'exercices produisent les mêmes effets dans toutes les parties du corps. La forme physique se borne à la force musculaire et à la mobilité (ils ne parlent jamais d'articulations) et le système cardio-vasculaire n'est pas nommé une seule fois. Les femmes ont moins de masse musculaire que les hommes et c'est pour cela qu'elles sont plus faibles, elles supportent beaucoup moins l'effort physique. Une femme haltérophile développera énormément ses muscles et aura un air masculin. Les élèves ne connaissent pas la fonction de la testostérone dans le développement des muscles de l'homme. Dans le cas des lésions, d'un joueur de football par exemple, ces élèves l'associent à la notion de malheur, de fatalité.

\subsection{Santé et Environnement}

17 Par rapport à l'environnement et à la santé, nous constatons l'usage d'expressions qui sont en correspondance avec les schémas conceptuels, avec les valeurs et les croyances des sociétés où l'on vit.

Il y a des concepts tels que progrès, développement, solidarité, qui sont mêlés à d'autres concepts. Il faut considérer que si l'étude de ces concepts n'est pas introduite dans l'éducation pour l'environnement, ils seront traités plus difficilement dans d'autres matières. La désertification, l'effet de serre, les résidus solides urbains, sont nommés par les élèves sans aucune cohérence, isolés d'autres concepts préalables compréhensibles et fondamentaux. Apparemment, ils rapportent ce que disent les medias, par effet de mode et influence de l'écologisme politique. 
19 La probabilité que des élèves construisent des connaissances scientifiques, même s'il s'agit de problèmes qui leur sont proches est très réduite; peut-être parce que ces concepts sont très éloignés de leur perception immédiate, parce qu'ils sont reconnus comme des informations des professeurs qui leur apportent « les affaires découvertes par les scientifiques ».

\subsection{Drogues}

20 L'alcool est la drogue la plus ancienne qu'on connaît, mais les jeunes ne le savent pas encore et ils ne le considèrent pas comme une drogue. Ils croient qu'une personne qui boit est capable de s'arrêter quand elle le veut. Les effets et les conséquences de l'alcool sur l'état physique ne sont pas des sujets très clairs pour ces étudiants: les troubles gastro-intestinaux (diarrhée) sont les seuls qu'ils nomment comme effet immédiat de l'alcool. Ils ne connaissent pas les maladies neurologiques causées par la consommation d'alcool (insomnie, cauchemars, troubles psychomoteurs allant même jusqu'aux crises convulsives, psychose alcoolique et delirium tremens). À propos des troubles sexuels conséquents de la consommation d'alcool, les élèves affirment avoir entendu parler de l'impuissance de l'homme, mais ils pensent que cela n'arrive qu'aux hommes âgés (après quarante ans). En plus, ils confondent les termes impuissance et éjaculation précoce. Dans certains cas, on trouve l'idée que le Syndrome de Down peut être le résultat des effets de l'alcool dans le sang du père ou de la mère.

Ils considèrent comme seules drogues celles qui ne sont pas institutionnalisées (le cannabis, les colles ou les solvants, la cocaïne, l'héroïne, les amphétamines, etc.). Leurs effets physiques et psychologiques ne sont pas connus. Bien qu'ils disent connaitre les effets psychologiques, au moment d'être interrogés sur les effets des drogues sur le système nerveux ils parlent de la "déprime » (dépression), mais ils ne savent pas clairement ce qu'il se passe au niveau du système nerveux.

Ils considèrent le tabac comme une cause des maladies de l'appareil respiratoire et même du cancer du poumon, mais ils pensent aussi que le fait d'avoir un cancer du poumon est en relation avec d'autres facteurs tels le malheur ou l'hérédité d'un parent très proche.

\subsection{M.S.T. et SIDA}

23 À propos des maladies sexuellement transmissibles, la grande majorité d'élèves entre 9 et 18 ans montrent franchement une ignorance de certaines maladies (syphilis, gonorrhée, herpès génital et chlamydia). Ils répondent qu'ils n'en ont jamais entendu parler ou qu'ils ne savent pas trop de quoi il s'agit. Ceux qui croient savoir quelque chose n'ont pas d'informations sûres. Par exemple, à propos de la syphilis, quand on leur demande s'il est vrai ou faux qu'un individu peut être de nouveau contaminé, ou si l'on peut être infecté par une personne ne présentant pas de symptômes, la majorité des étudiants répond «je ne sais pas".

Par rapport au SIDA, la situation est assez différente. Beaucoup d'élèves croient savoir certaines choses sur cette maladie. Quand ils sont interrogés plus profondément, cependant, on constate qu'ils présentent d'importantes lacunes informatives sur cette maladie et des opinions erronées, bien que la grande majorité connaisse quelques mécanismes de transmission et quelques mesures préventives. Ils affirment aussi ne pas 
savoir comment agit le HIV ou ce qu'est un porteur asymptomatique, ne pas avoir des renseignements sur le système immunitaire et les processus de l'infection.

\section{Concepts biologiques nécessaires}

Dans le précédent paragraphe nous avons pointé l'existence d'un savoir acquis enfantin, intuitif, préscientifique, et le plus souvent lié à des croyances traditionnelles, transmises par le milieu et la famille. La connaissance de ces conceptions est fondamentale pour y adapter les stratégies d'enseignement-apprentissage et les mesures de promotion de la santé.

À notre avis, pour atteindre une amélioration des connaissances des élèves, il est nécessaire, entre autre, de développer une base conceptuelle préalable.

\subsection{Alimentation et Nutrition}

Énergie et transformations énergétiques :

- bilan énergétique du métabolisme

- concept d'énergie

Composition des aliments (fondements biochimiques et physiologiques) :

- concepts physico-chimiques

- différenciation entre aliments plastiques et énergétiques

- différenciation entre aliment et nutriment

Processus physiologique de la nutrition (au niveau des cellules)

Notions de diététique

- classement selon leur fonction :

- bilan énergétique

- diète équilibrée

- contrôle du poids

\subsection{Activité physique}

Anatomie, physiologie et biomécanique :

- fonctionnement du corps et les effets de l'exercice

- habitudes et mécanique du mouvement

- fonctions du coeur (circulation du sang)

- fonctions respiratoires (mécanique de la respiration, rôle du poumon

- dans les excrétions)

- force, vélocité, résistance - effets sur les femmes

Problématique de l'exercice physique et ses rapports avec l'état de santé de l'individu :

- différence entre sport, activité physique ou exercice physique

\subsection{Santé et Environnement}

Concept d'Écologie ;

Concept d'environnement, milieu naturel, historique, culturel, socio-économique et 
technologique ;

Protection de la nature - polluants, désertification, effet de serre ;

Développement soutenu et industrialisation.

\subsection{Drogues}

Physiologie :

- système gastro-intestinal, neurologique, génital et immunologique

Conséquences physiques et psychiques de l'alcool :

- troubles gastro-intestinaux

- intoxication alcoolique

\subsection{M.S.T. et SIDA}

Système immunitaire :

- fonctionnement

- rapport antigène et anticorps

Infections par virus, bactéries et champignons :

- infections opportunistes

Concepts de transmission et contagion

\section{Conclusion}

Finalement, on constate que les curricula écoliers en Espagne, même dans le processus de la Réforme de l'Enseignement, ne sont pas conçus de façon convenable pour le développement de ce genre d'éducation. Des essais de "sujets transversaux » (la santé, la consommation, l'environnement, etc.) intégrés dans les différents domaines du savoir des curricula écoliers sont tentés. Néanmoins, cette idée n'est pas facile à mettre en pratique dans les situations normales d'une école; de plus, cette approche conduit à traiter ces sujets de façon optionnelle, sans connexion avec l'énoncé plus académique du reste du curriculum.

Il s'en suit, de ce point de vue, qu'on doit exiger un changement dans les stratégies d'enseignement des clichés d'EAS. Les élèves peuvent arriver à acquérir des connaissances de façon mécanique et ils admettront peut-être comme valables quelques informations mais, sans la base conceptuelle biologique adéquate, ces informations ne parviendront pas à produire un changement de leurs idées préalables. Il n'y aura pas d'apprentissage significatif et on ne pourra donc pas arriver au principal objectif d'une éducation pour la santé : la modification des attitudes et des conduites des élèves face à leur propre santé. 
Notez la rareté des détails concernant les microbes et la censure portant sur l'anatomie génitale masculine.

p. 276 : Figure 16 et Figure 17.

LORULOTA., La Véritable ÉDUCATION SEXUELLE - Bréviaire complet de la Santé et du Bonheur en Amour, Paris, Éditeur : Paris-Édition, 1930 - Copyright : Georges An-quetil, 1926.

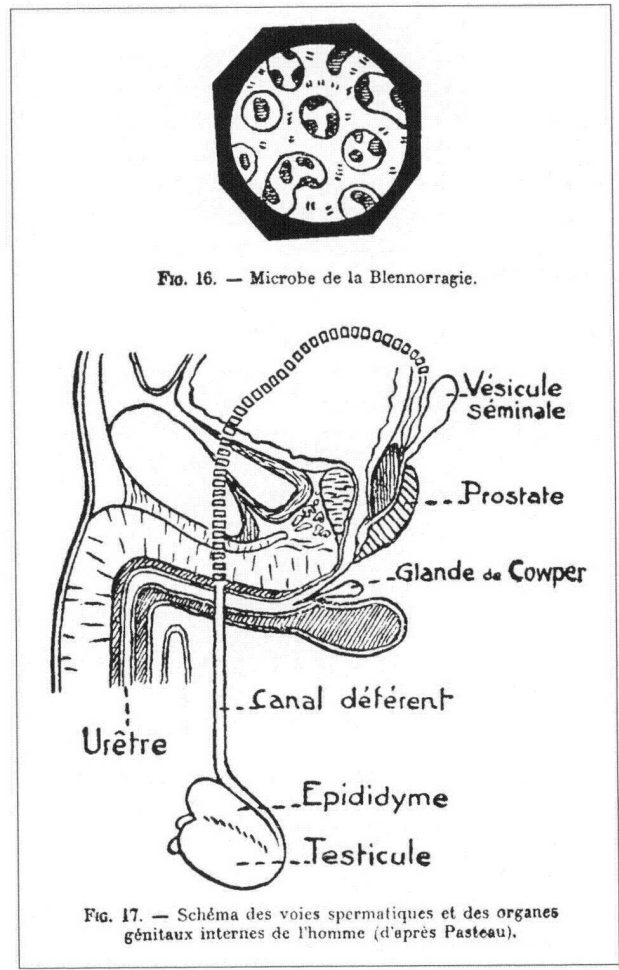

\section{BIBLIOGRAPHIE}

AUSUBEL D.P., Psicología Educativa. Un punto de vista cognoscitivo, México, Trillas, 1978.

BANET E. et al., «Ideas de los alumnos sobre digestió : aspectos anatómicas », in Enseñanza de las Ciencias, Vol. $1: \mathrm{N}^{\circ} 6,1988$, p. 30-37.

BANET, E. et al., « Ideas de los alumnos sobre digestió : aspectos fisiológicas », in Enseñanza de las Ciencias, Vol. 1 : $\mathrm{N}^{\circ} 7,1989$, p. 35-44.

CUBERO R., " Estudio de los esquemas de conocimiento relativos al proceso digestivo ", in IV Jor. Est. Invest. Escuela, Sevilla, 1986, p. 139.

DRIVER R., « Un enfoque constructivista para el desarrollo del curriculum de ciencias », in Enseñanza de las Ciencias, $\mathrm{N}^{\circ}$ 6, 1986, p. 109-120.

FERNANDES A. et MANZANARES, M., Pupils health idea, Genève, sous presse, 1995. 
GIL D. et al., « Tres paradigmas básicos en la Enseñanza de las Ciencias », in Enseñanza de las Ciencias,Vol. 1 : N 1, 1985.

GIORDAN A., Conceptos de Biología : tomos I y II. Barcelona, Labor, 1987.

MINTZE, J.J., "Naive Theories in Biology : children's concepts of the Human Body", in Scholl Science and Mathematics. Vol. 7 : N84, 1984, p. 548-555.

NOVAK J.D. Teoría y práctica de la Educación. Madrid. Alianza Universidad. 1982. 275 p.

POSNER G.J., "Accomodation of a scientific conception : towards a theory of conceptual change", in Science Education, $\mathrm{N}^{\circ}$ 66, 1982, p. 211-227.

PROUT A., "Science, health and everyday knowledge : a case study about the common cold", in European Journal of Science Education. Vol. 4 : N 7, 1985, p. 399-406.

YUS R., Investigando la naturaleza (2 ${ }^{\text {ndo }}$ curso de E.S.O.), Guía del Alumno y del Profesor, Velez-Màlaga. Elzevir, 1991.

\section{RÉSUMÉS}

Ce travail résulte d'une réflexion et d'une analyse des préconceptions des élèves sur la santé. Actuellement, nous révisons nos propres contenus méthodologiques qui touchent notamment au rôle des concepts scientifiques sous-jacents dans les processus d'enseignement - apprentissage. Cela nous conduit à mettre en évidence les idées préalables de certains élèves et à proposer des concepts biologiques pour introduire les notions souhaitées.

This research work is the result of a reflection and of an analysis about students' preconceptions on health. At the present time, we are revising our own methodological contents, especially inasmuch as they touch upon the role of the underlying scientific concepts underpinning the various teaching and learning processes. This leads us to bring to the fore the previously held ideas of certain students and to put forward various biology concepts so as to introduce the notions one wishes to teach.

\section{INDEX}

Mots-clés : apprentissage significatif, éducation pour la santé, préconception

Keywords : health education, preconception, significant learning process

\section{AUTEURS}

\section{ALIANA FERNANDES-GUIMARÄES}

Departemento de Didactica de las Ciencias Sociales y Experimentales, universidad de Cordoba (Espana)

\section{MERCEDES MANZANARES-GAVILAN}

Departemento de Didactica de las Ciencias Sociales y Experimentales, universidad de Cordoba (Espana) 